\title{
Intensivtherapie bei schwerer Pankreatitis
}

\author{
E. MARTIN und H. SCHMIDT
}

Die akute Pankreatitis stellt ein Krankheitsbild mit einem breiten Spektrum klinischer Erscheinungsformen dar. Schätzungsweise $80 \%$ der akuten Episoden zeigen einen milden, selbstlimitierenden Verlauf. Das pathomorphologische Bild ist hierbei durch ein interstitielles Ödem des Pankreas mit minimalen Fettgewebsnekrosen gekennzeichnet. Diese Patienten zeigen unter einer supportiven Basistherapie ohne spezifische Behandlungsverfahren eine raschen Rückgang der klinischen Symptomatik [1]. Die bei 5-20\% aller Pankreatitiden auftretende schwere, nekrotisierende Verlaufsform ist aufgrund der assoziierten renalen, pulmonalen und hämodynamischen Komplikationen durch eine hohe Letalität gekennzeichnet [2]. Morbidität und Letalität der nekrotisierenden Pankreatitis werden von der Wirkung vasoaktiver und toxischer Substanzen, der Ausdehnung von Fettgewebs- und Parenchymnekrosen sowie einer sekundären bakteriellen Kolonisation des nekrotischen Materials bestimmt [3]. Diese Tatsache findet ihren Niederschlag in den verschiedenen Therapieansätzen der akuten nekrotisierenden Pankreatitis. Die Spannbreite der Behandlungsmodalitäten reicht hierbei von ausschließlich konservativen Maßnahmen $[4,5]$ bis hin zu frühzeitigen ausgedehnten operativen Eingriffen mit dem Ziel der Entfernung von nekrotischen, devitalisiertem Material [6,7]. Eine spezifische therapeutische Interventionsmöglichkeit existiert trotz erweiterter Einblicke in die Pathophysiologie der akuten Pankreatitis bislang nur in Ansätzen. Die Senkung der Letalität der nekrotisierenden Pankreatitis auf 15-20\% [1] beruht nicht zuletzt auf einer konsequenten Intensivtherapie systemischer Komplikationen während des gesamten Krankheitsverlaufes. Im folgenden sollen intensivmedizinische Strategien zur Behandlung der nekrotisierenden Pankreatitis aufgezeigt und diskutiert werden.

\section{Definition}

Eine akute abdominelle Schmerzsymptomatik stellt das klinische Leitsymptom der akuten Pankreatitis dar. Laborchemisch führend ist die Erhöhung der Pankreasenzyme Amylase und Lipase in Serum und Urin sowie eine Hypokalziämie. Nach der revidierten Klassifikation von Marseille [8] wird die Pankreatitis in eine leichte und eine schwere Verlaufsform eingeteilt. Pathomorphologisch findet sich bei der leichteren interstitiellödematösen Form ein generalisiertes Pankreasödem mit vereinzelten Fettgewebsnekrosen. Die schwere, nekrotisierende Pankreatitis ist durch ausgedehnte peri- und intrapankreatische Fettgewebsnekrosen, Parenchymnekrosen und Hämorrhagien gekennzeichnet [9]. Als weitere Klassifikation der akuten Pankreatitis hat sich die in Tabelle 1 dargestellte pathomorphologische Einteilung durchgesetzt. Hierbei stellen der Pankreasabszeß und die Pankreaspseudozyste Spätkomplikationen der akuten Pankreatitis dar.

\section{Ätiologie und Pathogenese der akuten Pankreatitis}

Ätiologie und Pathogenese der akuten Pankreatitis sind außerordentlich komplex. Bei der Ätiologie der akuten Pankreatitis (Abb. 1) spielen die biliäre und die alkoholtoxische Genese die führende

Tabelle 1. Pathomorphologische Einteilung der Pankreatitis

\begin{tabular}{|c|c|}
\hline Pathomorphologie & $\begin{array}{l}\text { Häufigkeit } \\
{[\%]}\end{array}$ \\
\hline Interstitiell-ödematöse Pankreatitis & $70-80$ \\
\hline Nekrotisierende Pankreatitis & $10-20$ \\
\hline Pankreasabszeß & 2 \\
\hline Pseudozyste & $3-5$ \\
\hline
\end{tabular}




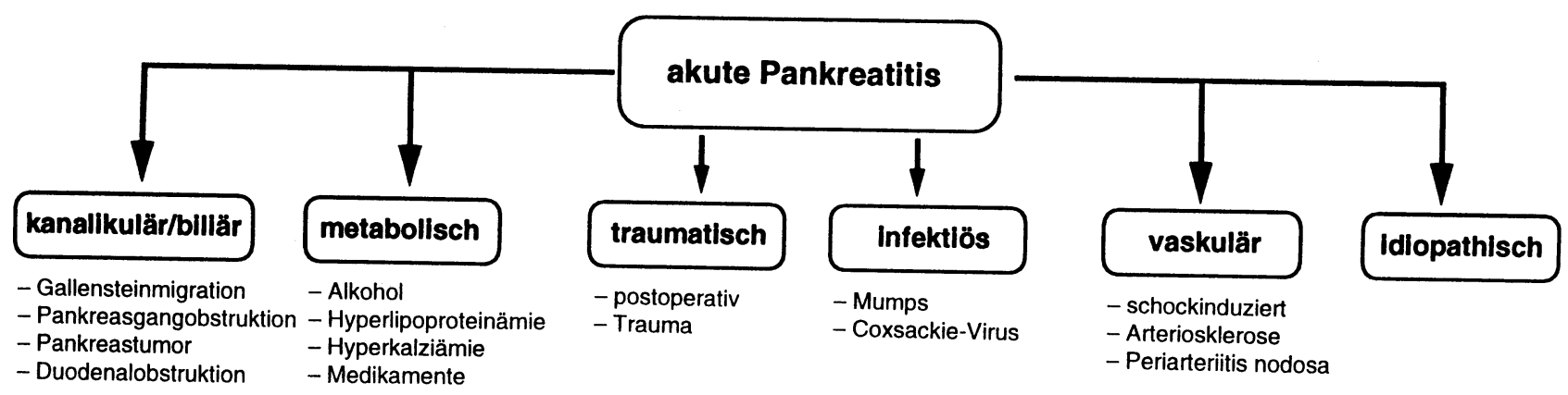

Abb. 1. Ätiologie der akuten Pankreatitis

Rolle. Seltener sind die metabolische, traumatische, infektiöse, vaskuläre und idiopathische Genese. Ätiologisch dominierend in Europa ist mit 40-50\% die alkoholtoxische Pankreatitis, gefolgt von der biliären Pankreatitis (30-40\%). Bei ca. $20 \%$ der akuten Pankreatitiden ist keine klare ätiologische Zuordnung möglich; ca. 5\% werden durch Stoffwechselstörungen verursacht. Das wesentliche pathogenetische Grundprinzip besteht in einem Austritt lytischer Enzyme in das Interstitium, einer Aktivierung der Enzymsysteme sowie einer Mikrozirkulationsstörung des Pankreas. Bei der biliären Pathogenese der akuten Pankreatitis stellt die Migration von Gallensteinen mit einer passageren Obstruktion im Bereich der Papille den pathogenetisch entscheidenden Faktor dar. Aus der gallensäureninduzierten Steigerung der Gangpermeabilität kombiniert mit einer intraduktalen Druckerhöhung und Austritt von aktivierten Pankreasenzymen in das Interstitium resultiert die Entwicklung eines Pankreasödems [10]. Bei der Pathogenese der Alkoholpankreatitis - spielen sowohl ein Refluxobstruktionsmechanismus als auch eine direkte zytotoxische Wirkung des Alkohols eine Rolle. Die akute Ingestion von Alkohol führt zu einer Stimulation der Pankreassekretion und zu einer Tonuszunahme des Sphinkter Oddi; bei chronischer Alkoholaufnahme ist eine erhöhte Proteinpräzipitation mit rezidivierendem Sekretstau beschrieben [11]. Weiterhin wird durch Alkohol eine generalisierte Permeabilitätsstörung des Pankreas mit Übertritt lytischer Enzyme in das Interstitium induziert [12]. Infolge dieser Enzymaktivierung im Interstitium kommt es zu einer Autodigestion des Pankreas.

Ein ebenfalls diskutierter intraazinärer Ausgangspunkt der akuten Pankreatitis beruht auf einer basolateralen, interstitiellen Sekretion von Pankreasenzymen sowie einer intrazellulären $\mathrm{Fu}$ sion von Zymogengranula und Liposomen. Durch diese Fusion kommt es zu einer intrazellulären Trypsinaktivierung im sauren Milieu der Lysosomen [10].

Der entscheidende Mechanismus beim Übergang von der interstitiell-ödematösen Pankreatitis zur nekrotisierenden Pankreatitis scheint die Störung der Mikrozirkulation des Pankreas darzustellen [10]. Als ursächlich hierfür werden eine Vasokonstriktion, eine direkte Schädigung des Gefäßendothels infolge interstitieller Gallensäuren und Trypsin, freie $\mathrm{O}_{2}$-Radikale infolge ischämie-induzierter Aktivierung der Xanthinoxidase, eine durch Trypsineinschwemmung induzierte intravasale Gerinnung und eine durch erhöhten interstitiellen Druck bei Plasmasequestrierung verursachte venöse Obstruktion diskutiert [10]. Zusammenfassend lassen sich als pathogenetische Voraussetzungen für die Entstehung einer akuten Pankreatitis der interstitielle Austritt von Pankreasenzymen, ihre Aktivierung im Interstitium sowie eine Störung der pankreatischen Mikrozirkulation nennen (Abb. 2).

\section{Klinik der akuten Pankreatitis}

Klinisches Leitsymptom der akuten Pankreatitis ist der akute abdominelle Schmerz mit Lokalisation im Oberbauch und gürtelförmiger Ausstrahlung in den Rücken, der nach schleichendem Beginn nach Stunden sein Maximum erreicht. Der abdominelle Palpationsbefund reicht von einem lokalisierten Druckschmerz bis hin zur generalisierten Abwehrspannung. Die Allgemeinsymptomatik ist geprägt von einer Dehydration, deren Ausmaß von einer diskreten Hypovolämie bis zum manifesten Volumenmangelschock reicht. Weiterhin können eine Reihe systemischer und lokaler Komplikationen auftreten [13]. (Tabelle 2). Die bei $80 \%$ der Patienten auftretende interstitiell- 
Abb. 2. Pathogenese der akuten Pankreatitis

ödematöse Pankreatitis unterscheidet sich von der nekrotisierenden Pankreatitis nicht durch die Schmerzintensität und -charakteristik, sondern durch das Fehlen sekundärer Komplikationen. Auch bei der nekrotisierenden Pankreatitis ist die Frühletalität gering; ein foudroyanter letaler Verlauf ist nur selten zu beobachten. Todesursache ist überwiegend ein Multiorganversagen als Folge septischer Komplikationen im Verlauf von einigen Wochen. Typischerweise ist die nekrotisierende Pankreatitis in ihrem Verlauf durch 2 Phasen gekennzeichnet. In einer frühen toxischen Phase kommt es bei einem generellen Entzündungsprozeß im Sinne eines SIRS (,systemic inflammatory response syndrome“) zu ausgedehnten Flüssigkeitsverschiebungen, interstitieller Ödembildung sowie einer hämodynamischen, respiratorischen und renalen Insuffizienz. Als auslösend hierfür werden verschiedene pankreatogen getriggerte Mediatorsysteme (Interleukine, Komplementsystem, Lipoxygenase-, Cyclooxygenasesystem) der allgemeinen Entzündungsreaktion angesehen. Die in ihrer Häufigkeit führende pulmonale Insuffizienz beruht hauptsächlich auf Gasaustauschstörungen infolge eines durch erhöhte Kapillarpermea-

Tabelle 2. Komplikationen bei akuter Pankreatitis

\begin{tabular}{ll}
\hline Komplikationen & $\begin{array}{l}\text { Häufigkeit } \\
{[\%]}\end{array}$ \\
\hline Volumenmangelschock & 11 \\
ARDS & 19 \\
Niereninsuffizienz & 15 \\
Gastrointestinale Blutung & 15 \\
\hline
\end{tabular}

bilität auftretenden interstitiellen Ödems [14]. Hämodynamische Veränderungen bei akuter Pankreatitis entsprechen denen des septischen Schocks und sind durch ein erhöhtes Herzzeitvolumen, einen niedrigen arteriellen Mitteldruck und niedrige systemische Widerstände gekennzeichnet $[15,16,17]$.

Die 2., späte Phase der nekrotisierenden Pankreatitis ist durch die sekundäre bakterielle Besiedelung avitaler Nekrosenbezirke und der daraus sich ergebenden Komplikationen gekennzeichnet. Neben der Bildung lokalisierter Abszesse und infizierter Nekrosen kommt es zu generalisierten septischen Komplikationen und zum Multiorganversagen [18]. Der klinische Verlauf der akuten Pankreatitis ist in Abb. 3 dargestellt.

\section{Diagnostik}

Zur Sicherung der klinischen Verdachtsdiagnose akute Pankreatitis werden laborchemische und bildgebende Verfahren eingesetzt (Abb. 4). Ziel der differentialdiagnostischen Abklärung ist die Sicherung der Verdachtsdiagnose und die Festlegung von Ausmaß und Schweregrad der Erkrankung. Eine Erhöhung der Pankreasenzyme Amylase und Lipase im Serum unterstützt die Diagnose Pankreatitis, jedoch ist die Höhe der Enzymkonzentrationen kein sicherer Indikator für den Schweregrad oder die Prognose einer akuten Pankreatitis. Differentialdiagnostisch sind andere Erkrankungen, die eine Hyperamylasämie oder eine Hyperlipasämie verursachen, auszuschließen. Für die Diskriminierung zwischen ödematös-inter- 
Abb. 3. Klinischer Verlauf der akuten Pankreatitis

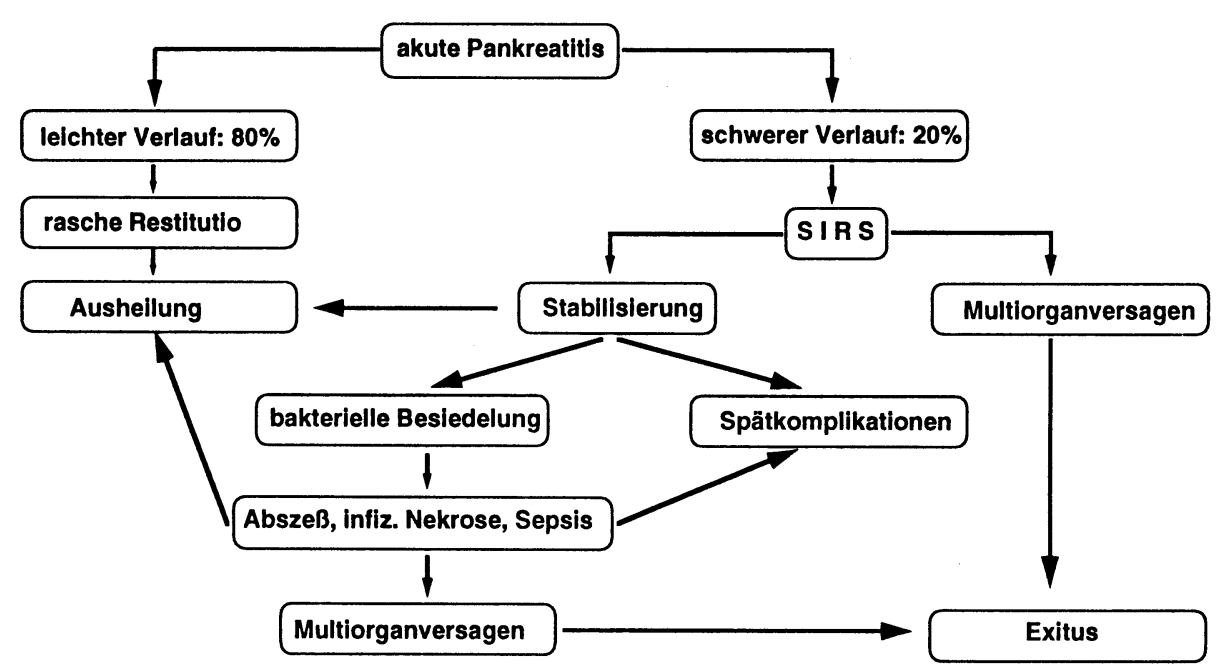

stitieller Pankreatitis und nekrotisierender Pankreatitis haben neben den Parametern $\alpha_{1}$-Antitrypsin und $\alpha_{2}$-Makroglobulin v.a. eine Erhöhung von LDH und CRP einen diagnostischen Stellenwert. So korreliert eine LDH-Erhöhung in $87 \%$ und eine CRP-Erhöhung in 93\% der Fälle mit einer Nekrosebildung im Verlauf einer Pankreatitis [19]. Eine sehr enge Korrelation besteht zwischen dem Nekroseausmaß und der Prognose einer Pankreatitis einerseits und dem Spiegel von Trypsinogenaktivationspeptiden (TAP) andererseits [20]. Diese
Peptide werden bei der Aktivierung von Trypsinogen zu Trypsin abgespalten und freigesetzt. Normalerweise werden diese Peptide rasch von der Darmflora neutralisiert. Bei intrapankreatischer Trypsinaktivierung werden diese Peptide jedoch systemisch absorbiert und können deshalb im Serum nachgewiesen werden. Prognostische Bedeutung für das Outcome des Patienten haben auch andere Laborwerte wie Serumkalzium, Blutgase, harnpflichtige Substanzen und Transaminasen [21].

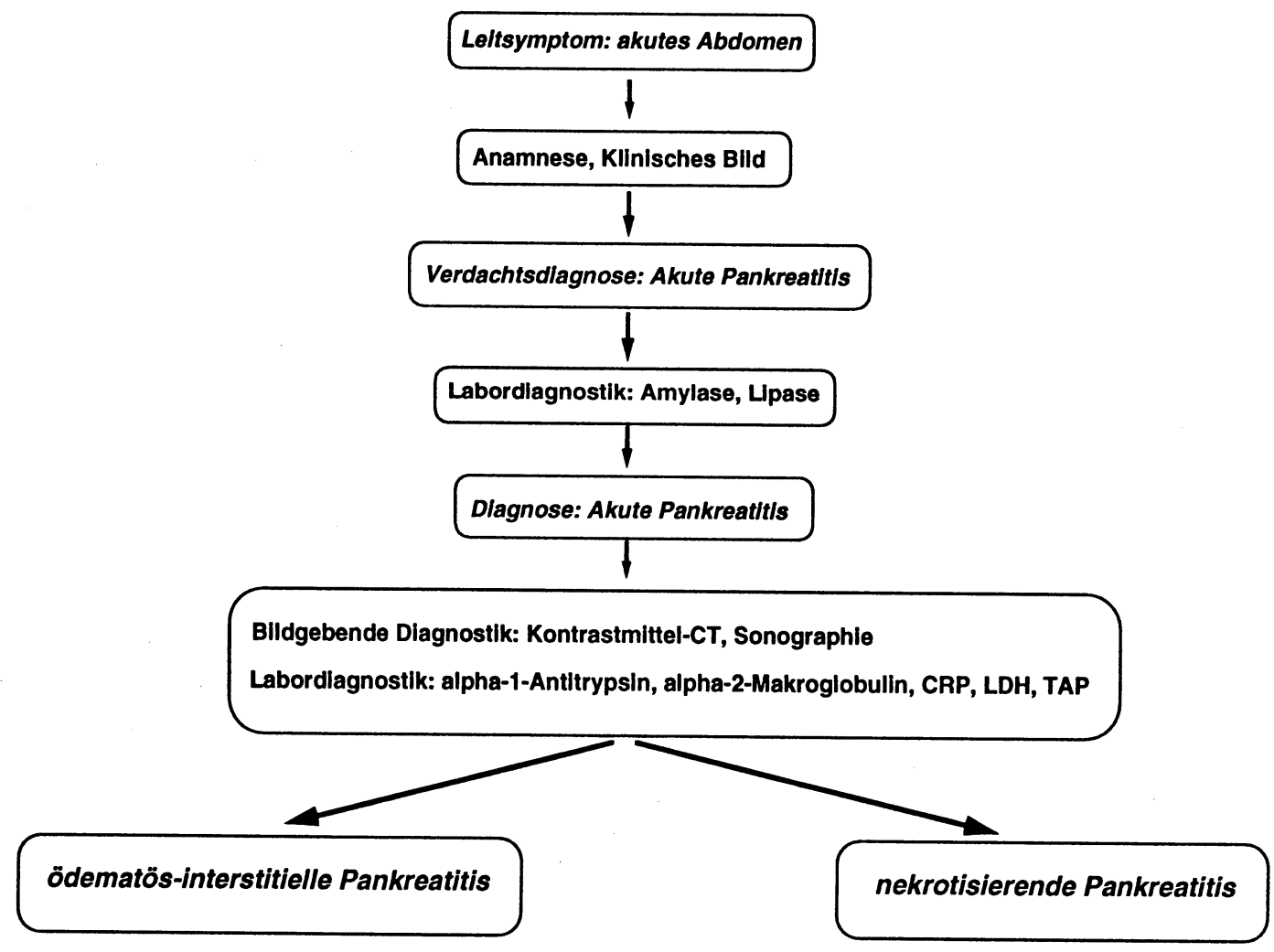

Abb. 4. Diagnostik bei Verdacht auf Pankreatitis 
Tabelle 3. Diagnostische Frühkriterien der Pankreatitis nach Ranson und Imrie

\begin{tabular}{|c|c|}
\hline $\begin{array}{l}\text { Akute Pankreatitis } \\
\text { (Ranson) }\end{array}$ & $\begin{array}{l}\text { Akute Pankreatitis } \\
\text { (Imrie) }\end{array}$ \\
\hline Bei Aufnahme: & Bei Aufnahme: \\
\hline $\begin{array}{l}\text { Alter }>55 \mathrm{Jahre} \\
\text { Leukozytenzahl } 16000 / \mathrm{mm}^{3} \\
\text { BZ }>200 \mathrm{mg} \% \\
\text { LDH }>350 \mathrm{IU} / 1 \\
\text { GOT }>250 \mathrm{U} / \mathrm{l}\end{array}$ & Alter $>55$ Jahre \\
\hline Nach 48 h: & Nach 48 h: \\
\hline HKT-Abfall > $10 \%$ & Leukozytenzahl $>15000 / \mathrm{mm}^{3}$ \\
\hline $\begin{array}{l}\text { Anstieg des Harnstoff-N } \\
\text { um mehr als } 5 \mathrm{mg} \%\end{array}$ & $\begin{array}{l}\text { Harnstoff }>16 \mathrm{mmol} / \mathrm{l} \\
\text { Kreatinin }>16 \mathrm{mmol} / \mathrm{l}\end{array}$ \\
\hline Serumkalzium $<2 \mathrm{mmol} / \mathrm{l}$ & Serumkalzium $<2 \mathrm{mmol} / \mathrm{l}$ \\
\hline Basendefizit $>-4 \mathrm{mmol} / \mathrm{l}$ & $\mathrm{BZ}>200 \mathrm{mg} \%$ (kein Diabetiker) \\
\hline Flüssigkeitsretention $>61$ & Albumin $<32 \mathrm{~g} / 1$ \\
\hline $\mathrm{p}_{\mathrm{a}} \mathrm{O}_{2}<60 \mathrm{~mm} \mathrm{Hg}$ & $\begin{array}{l}\mathrm{p}_{\mathrm{a}} \mathrm{O}_{2}<60 \mathrm{~mm} \mathrm{Hg} \\
\text { GOT/GPT }>100 \mathrm{U} / \mathrm{l}\end{array}$ \\
\hline
\end{tabular}

Zur bildgebenden Standarddiagnostik der akuten Pankreatitis zählen die transabdominelle Sonographie und die Computertomographie. Während mit Hilfe der Sonographie nur in ca. 50\% der Fälle aufgrund des Begleitmeteorismus eine Darstellung und Beurteilung der Pankreasloge und des Retroperitonealraumes gelingt [22], ist die Kontrastmittelcomputertomographie z.Z. als diagnostische Referenzmethode anzusehen. So zeigt die Computertomographie eine Sensitivität von $99 \%$ und eine Spezifität von $100 \%$ in der Diagnostik der akuten Pankreatitis [23]. Beurteilungsparameter sind v.a. Größe, Kontur und Homogenität des Organes sowie extrapankreatische Flüssigkeitsansammlungen und Nekrosestraßen. Durch wiederholte CT-Untersuchungen können auch Komplikationen wie Nekrosenbildung, Abszesse und Pseudozysten mit großer Sicherheit erkannt werden. Insbesondere in der Festlegung der therapeutischen Strategie zwischen konservativer Intensivtherapie und operativer Intervention bei Ausbildung lokaler Komplikationen ist die Computertomographie von hohem Stellenwert. Nachteilig ist jedoch die potentielle Möglichkeit einer weiteren Schädigung des Pankreas durch das verwendete Kontrastmittel.

Zur klinischen Einschätzung des Schweregrades einer Pankreatitis werden verschiedene Score- systeme verwendet. Am häufigsten werden z.Z. das Ranson-System [21, 24] und das Imrie-System [25] eingesetzt (Tabelle 3). Diese können im frühen Verlauf einer Pankreatitis angewendet werden, wobei das Vorhandensein von 3 oder mehr positiven Faktoren auf einen schweren Verlauf hindeuten. Nachteilig bei beiden Scoresystemen ist jedoch, daß der komplette Scorewert erst $48 \mathrm{~h}$ nach Aufnahme vorliegt.

\section{Intensivmedizinische Strategien der Behandlung der schweren Pankreatitis}

Die Therapieprinzipien bei schwerer akuter Pankreatitis umfassen ein aktives Monitoring, eine suffiziente Schmerztherapie, die Ruhigstellung des Pankreas sowie spezifische intensivmedizinische und operative Maßnahmen je nach Schweregrad und Stadium der Erkrankung. Alle Patienten mit der Verdachtsdiagnose Pankreatitis sollten auf Intensivstationen zur Überwachung der Vitalfunktionen aufgenommen werden. Kardiozirkulatorischer Schock, akutes respiratorisches Versagen, renale Insuffizienz und eine metabolische Entgleisung entwickeln sich im Frühstadium der Erkrankung (toxische Phase) und erfordern eine sofortige therapeutische Intervention. 
Tabelle 4. Basistherapie der schweren Pankreatitis

\begin{tabular}{lll}
\hline Ruhigstellung des Pankreas & $\rightarrow \quad \begin{array}{l}\text { Nahrungskarenz, } \\
\text { nasogastrale } \\
\text { Ableitung }\end{array}$ \\
Schmerztherapie & $\rightarrow \quad \begin{array}{l}\text { Procaindauerinfusion }(2 \mathrm{~g} / \mathrm{Tag}), \\
\text { Buprenorphin, Tramadol, } \\
\text { Periduralanästhesie }\end{array}$ \\
Hämodynamische Stabilisierung & $\rightarrow \quad \begin{array}{l}\text { Volumentherapie: } \\
\text { kristalloide/kolloide Lösungen (3 1/Tag); } \\
\text { medikamentös: }\end{array}$ \\
Katecholamine (Noradrenalin, Adrenalin) \\
Respiratorische Stabilisierung & $\rightarrow \quad \begin{array}{l}\text { On-Applikation, } \\
\text { kontrollierte Beatmung + PEEP + IRV }\end{array}$ \\
Therapie der Niereninsuffizienz & $\rightarrow \quad \begin{array}{l}\text { Volumentherapie, } \\
\text { Verbesserung der Nierenperfusion (Dopamin), } \\
\text { Diuretika, } \\
\text { Hämofiltration } \\
\text { frühzeitige parenterale Ernährung }\end{array}$ \\
& &
\end{tabular}

\section{Basistherapie}

Die Basistherapie (Tabelle 4) der akuten Pankreatitis besteht in einer Ruhigstellung des Pankreas durch orale Nahrungskarenz, in der nasogastralen Ableitung der Magenflüssigkeit bei paralytischem Ileus, in der Streßulkusprophylaxe mit $\mathrm{H}_{2}$-Antagonisten, Pirenzipin, Antazida oder Sucralfat, in einer adäquaten Volumensubstitution und in einer Normalisierung der Wasser-Elektrolyt- und SäureBasen-Haushaltes sowie in einer frühzeitigen parenteralen Ernährung [26]. Essentieller Bestandteil der Therapie ist eine suffiziente Analgesie, da die Schmerzen bei akuter Pankreatitis extrem ausgeprägt sein können. Morphin und Morphinderivate werden i.allg. aufgrund der potentiellen Tonussteigerung des Sphinkter Oddi vermieden, jedoch ist der Einsatz von Buprenorphin und Tramadol möglich [27]. Da tierexperimentelle Untersuchungen einen günstigen Einfluß von Procain auf die Letalität bei akuter experimentell erzeugter Pankreatitis gezeigt haben, wird zur Schmerzbekämpfung auch die kontinuierliche Infusion von Procainhydrochlorid $(2 \mathrm{~g} / 24 \mathrm{~h})$ eingesetzt [28]. Als weiteres schmerztherapeutisches Verfahren wird auch die epidurale Blockade in der Frühphase der Erkrankung propagiert [29]. Hierbei wird neben dem analgetischen Effekt auch ein spezifischer therapeutischer Effekt infolge der Sympathikusblockade und Verbesserung der Pankreasperfusion diskutiert.
Bei schwerer akuter Pankreatitis mit der Klinik eines SIRS ist ein erweitertes hämodynamisches Monitoring zur Steuerung der Volumentherapie notwendig. Eine direkte arterielle Blutdruckmessung sowie die Anlage eines Pulmonaliskatheters sind in diesem Falle indiziert.

Bei Patienten mit ödematös-interstitieller Pankreatitis ist eine am zentralen Venendruck und Urinausscheidung orientierte Volumentherapie mittels kristalloider Lösungen im allgemeinen ausreichend. Hierbei sollten anfangs mindestens 2,5-3 1 Elektrolytlösung/24 h infundiert werden, um die immer vorhandene Sequestrierung $\mathrm{zu}$ kompensieren [28]. Bei ausgeprägter Hypovolämie infolge einer erhöhten Kapillarpermeabilität und Flüssigkeitsverlusten in dritte Räume in der Frühphase einer nekrotisierenden Pankreatitis ist ein Volumenersatz mittels kolloidaler Lösungen vorteilhaft. Die Volumentherapie sollte sich dann an den mittels Pulmonaliskatheter ermittelten Größen „cardiac index“, pulmonalkapillärem Verschlußdruck sowie dem peripheren Gefäßwiderstand orientieren. Läßt sich mit Volumensubstitution kein ausreichender arterieller Mitteldruck ( $>70 \mathrm{~mm} \mathrm{Hg}$ ) erzielen, ist v.a. bei niedrigem peripherem Gefäßwiderstand eine $\alpha$ sympathomimetische Stimulation mit Noradrenalin indiziert.

Aufgrund der hohen Inzidenz einer pulmonalen Insuffizienz sollte die respiratorische Situation kontinuierlich mittels Pulsoxymetrie und inter- 
mittierend mittels Blutgasanalysen bereits in der Frühphase überwacht werden. Verschlechtert sich die respiratorische Situation trotz $\mathrm{O}_{2}$-Applikation, sollte frühzeitig die Indikation zur Intubation und kontrollierten Beatmung mit PEEP gestellt werden. Als orientierender Grenzwert zur Indikation einer Intubation kann ein Abfall des arteriellen $\mathrm{O}_{2}$-Partialdruckes auf Werte unter $60 \mathrm{~mm} \mathrm{Hg}$ angesehen werden.

Eine bereits im Frühstadium auftretende Oligurie kann ihre Ursache sowohl in einem prärenalen Nierenversagen infolge der Hypovolämie als auch in einem septisch-toxisch bedingten tubulär-interstitiellen Nierenversagen haben. Aus diesem Grund sollte bei beginnender Niereninsuffizienz das Flüssigkeitsregime angepaßt und die Nierenperfusion durch Applikation von Low-dose Dopamin $(2-5 \mu \mathrm{g} / \mathrm{kg} \mathrm{KG} / \mathrm{min})$ verbessert werden. Der Einsatz von Schleifendiuretika ist nur bei adäquater Volumensituation indiziert. Kommt es trotz diesen therapeutischen Maßnahmen nicht zu einer ausreichenden Diurese, ist v.a. bei gleichzeitiger respiratorischer Insuffizienz die frühzeitige Indikation zur kontinuierlichen Hämofiltration bzw. Hämodiafiltration zu stellen.

Die metabolischen Veränderungen bei schwerer Pankreatitis gleichen denen bei Sepsis und sind durch eine exzessive Katabolie gekennzeichnet. Die Kombination von Insulinresistenz und primärer entzündlich bedingter $\beta$-Zelldysfunktion resultiert in einer Hyperglykämie und Glukoseintoleranz. Eine negative Stickstoffbilanz bei schwerer
Pankreatitis ist mit einer erhöhten Letalität vergesellschaftet [30]. Ziel der Ernährungstherapie ist es, diese Katabolie zu durchbrechen. Eine Stimulation der exokrinen Pankreassekretion durch intragastrale und intraduodenale Stimuli muß vermieden werden. Hieraus ergibt sich die Forderung nach absoluter oraler Nahrungskarenz. Durch Applikation definierter Elementardiäten distal des Treitz-Bandes können die physiologischen gastropankreatischen und enteropankreatischen Sekretionsmechanismen jedoch umgangen werden, so daß eine enterale Ernährung über eine Katheterjejunostomie möglich ist [31]. Dies setzt jedoch einen operativen Eingriff voraus, der zumindest in der Frühphase der akuten Pankreatitis ohne Nachweis von Nekrosen oder Abszessen nicht indiziert ist. Als Standardernährungsregime hat sich die totale parenterale Ernährung durchgesetzt. Wie bei anderen intensivmedizinischen Krankheitsbildern erfolgt ein stufenweiser Aufbau der parenteralen Ernährung mit Glukose, Aminosäuren und Fetten bei engmaschigem Monitoring der Verträglichkeit der zugeführten Substanzen. Die Kohlenhydratzufuhr sollte im Bereich von $2-4 \mathrm{~g} / \mathrm{kg} \mathrm{Kg} / \mathrm{Tag}$ liegen, die Aminosäurensubstitution im Bereich von $1-1,5 \mathrm{~g} / \mathrm{kg} \mathrm{Kg} /$ Tag. Die parenterale Zufuhr von Fettemulsionen führt beim Menschen nicht zu einer Stimulation der exokrinen Pankreassekretion [32]. Auch konnte in zahlreichen Studien (Übersicht bei [31]) keine Exazerbation einer Pankreatitis durch intravenöse Fettapplikation gezeigt werden. Aus diesem Grund kann eine intravenöse

Tabelle 5. Spezifische konservative Therapiemaßnahmen bei schwerer Pankreatitis. (-) nicht effektiv; (+) experimentell: im Tierexperiment wirksam, klinischer Nachweis steht aus; (+) klinisch: klinische Hinweise auf Effektivität

\begin{tabular}{|c|c|c|c|}
\hline \multicolumn{3}{|l|}{ Spezifische Therapieansätze } & \multirow{2}{*}{$\begin{array}{l}\text { Wirksamkeit } \\
(-) \\
(-)\end{array}$} \\
\hline $\begin{array}{l}\text { Hemmung der intrapankreatischen } \\
\text { Enzymaktivierung }\end{array}$ & $\rightarrow$ & $\begin{array}{l}\text { Kallikreininhibitor } \\
\text { (Aprotinin), } \\
\text { Proteasen- + Phospholipase- } \text { A }_{2} \text {-Inhibitoren } \\
\text { (Gabexatmesilat, Camostat) }\end{array}$ & \\
\hline Hemmung der Pankreassekretion & $\rightarrow$ & $\begin{array}{l}\mathrm{H}_{2} \text {-Antagonisten, } \\
\text { Atropin } \\
\text { Kalzitonin, } \\
\text { Somatostatin, } \\
\text { Glukagon }\end{array}$ & $\begin{array}{l}(-) \\
(-) \\
(-) \\
(-) \\
(-)\end{array}$ \\
\hline Verbesserung der Mikrozirkulation & $\rightarrow$ & $\begin{array}{l}\text { isovolämische Hämodilution) } \\
\text { hyperonkotische Dextranlösungen }\end{array}$ & $\begin{array}{l}(+) \text { experimentell } \\
(+) \text { experimentell }\end{array}$ \\
\hline Mediatorelimination & $\rightarrow$ & kontinuierliche venovenöse Hämofiltration & (+) klinisch \\
\hline
\end{tabular}


Fettzufuhr bei Patienten mit nichthyperlipidämischer akuter Pankreatitis als sichere und effektive Form der Kaloriensubstitution angesehen werden [31]. Unter Monitoring des Triglyzeridspiegels ist deshalb eine intravenöse Zufuhr von Fettemulsionen in einer Dosierung von $1,5 \mathrm{~g} / \mathrm{kg}$ $\mathrm{Kg} / \mathrm{Tag}$ möglich.

\section{Spezifische Maßnahmen}

Die zunehmenden Kenntnisse über die der schweren Pankreatitis zugrundeliegenden pathophysiologischen Mechanismen haben zu einer Reihe von spezifischen Therapieansätzen geführt (Tabelle 5). Als im wesentlichen unwirksam haben sich hierbei die verschiedenen pharmakologischen Möglichkeiten der Blockierung aktivierter Pankreasenzyme sowie die pharmakologische Hemmung der exokrinen Pankreassekretion gezeigt. Neue Therapieansätze bestehen in der Verbesserung der Perfusion des Pankreas durch eine isovolämische Hämodilution und hyperonkotische Dextranlösungen sowie der Mediatorelimination mittels kontinuierlicher venovenöser Hämofiltration. Diese spezifischen Therapieverfahren sollen im folgenden besprochen werden.

\section{Hemmung der intrapankreatischen Enzymaktivierung}

Die Aktivierung autodigestiver Enzymsysteme wird als einer der pathogenetisch bedeutenden Faktoren in der Auslösung und Unterhaltung der akuten Pankreatitis angesehen. Aktiviertes Trypsin, Elastase, Prostaglandine und Phospholipase $\mathrm{A}_{2}$ sind in die Pathogenese der schweren Pankreatitis involviert. Eine direkte therapeutische Intervention durch spezifische Hemmung der intrapankreatischen Enzymaktivierung ist bisher jedoch nicht möglich. Es konnte weder durch den Kallikreininhibitor Aprotinin noch durch die synthetischen Proteasen- und Phospholipase- $\mathrm{A}_{2}$-Inhibitoren Gabexatmesilat (FOY) und Camostat (FOY 305) eine Senkung der Letalität der akuten Pankreatitis erreicht werden. Eine zunächst von Trapnell [33] beschriebene Verbesserung der Letalität von $25 \%$ in der Placebogruppe auf $7,5 \%$ in der Aprotiningruppe konnte in einer Multizenterstudie [34] nicht bestätigt werden, so daß der Einsatz dieses Präparates nicht mehr gerechtfertigt ist. Gleiches gilt für Gabexatmesilat und Camostat; auch hier konnte in einer multizentrischen Doppelblindstudie keine Senkung der Letalität erreicht werden [35]. Auch die Gabe natürlicher Serumantiproteasen in Form von Frischplasma zeigte keine signifikante Senkung der Letalität [36].

\section{Hemmung der Pankreassekretion}

Eine weitere theoretische Therapiemöglichkeit der akuten Pankreatitis besteht in der medikamentösen Hemmung der Pankreassekretion mit konsekutiver exokriner Ruhigstellung des Organes. Die indirekte Hemmung der Pankreassekretion durch $\mathrm{H}_{2}-$ Antagonisten hat sich jedoch nicht als effizient erwiesen [37], so daß diese nur zur Streßulkusprophylaxe eingesetzt werden. Atropin bewirkt zwar in niedrigen Dosierungen bereits eine ausgeprägte Hemmung der Pankreassekretion, zeigt jedoch keine günstige Wirkung auf den Verlauf einer akuten Pankreatitis [38].

Eine hormonelle Hemmung der Pankreassekretion kann durch Applikation von Glukagon, Kalzitonin und Somatostatin erreicht werden. Glukagon und Kalzitonin haben jedoch keinen signifikanten Effekt auf Letalität und klinischen Verlauf der akuten Pankreatitis [34, 39]. Auch für Somatostatin, das ebenfalls die exokrine Pankreassekretion hemmt, konnte in einer multizentrisch durchgeführten Studie kein Einfluß auf die Letalität gezeigt werden [40].

\section{Neue Therapieansätze}

Neue Therapieansätze bestehen in der Verbesserung der pankreatischen Mikrozirkulation durch isovolämische Hämodilution sowie in einer Toxinelimination mittels kontinuierlicher venovenöser Hämofiltration. Durch eine isovolämische Hämodilution mit Dextran 60 konnte tierexperimentell eine signifikante Verbesserung der Mikrozirkulation des Pankreas nachgewiesen werden [41]. Der zur Kapillarperfusion optimale Hämatokrit beträgt ca. $30 \%$. In einer weiteren tierexperimentellen Arbeit konnte der organprotektive Effekt hyperton-hyperonkotischer Lösungen demonstriert werden [42]. Die schnelle Bolusinfusion von Dextran 500000 mit oder ohne hypertone Kochsalzlösung (7,5\%) nach Induktion einer akuten Pankreatitis führte zu einer signifikanten Reduktion der Trypsinogenaktivierung, Nekrosebildung und Letalität. Der klinische Nachweis der 
Wirksamkeit dieser Therapie steht jedoch noch aus.

In der toxischen Phase der akuten Pankreatitis mit „systemic inflammatory response syndrome“ kommt es zu einer kaskadenartigen Aktivierung verschiedener Mediatorsysteme mit Konzentrationsänderungen von Bradykinin, Histamin, Prostaglandinen, Leukotrienen, Komplementfraktionen und Interleukinen. Interpretiert man diesen Zustand als Überschwemmung des Organismus mit Mediatoren und Zytokinen, so bietet sich die extrakorporale Elimination dieser Substanzen als nichtpharmakologischer Ansatz an. In einer retrospektiven klinischen Untersuchung bei Patienten mit septischem Multiorganversagen konnte eine Senkung der Letalität durch Hämofiltration demonstriert werden [43]. Inzwischen wurde von mehreren klinischen Arbeitsgruppen nachgewiesen, daß sowohl der Tumornekrosefaktor als auch das Interleukin-1 durch die kontinuierliche Hämofiltration eliminiert werden [44, 45]. Bei der Therapie der schweren nekrotisierenden Pankreatitis wurde durch frühzeitigen Einsatz der kontinuierlichen venovenösen Hämofiltration und ausschließlich konservativer Therapie eine deutliche Senkung der Letalität beschrieben [46].

\section{Zusammenfassung}

Entscheidend für eine differenzierte Therapie der akuten Pankreatitis ist die möglichst frühzeitige und sichere Unterscheidung zwischen der ödematös-interstitiellen und der nekrotisierenden Verlaufsform mittels bildgebender (Sonographie, Kontrastmittelcomputertomographie) und laborchemischer Verfahren (Amylase, Lipase). Je nach Schweregrad der akuten Pankreatitis sind differenzierte diagnostische und therapeutische Maßnahmen einzuleiten. Da eine spezifische therapeutische Intervention in das pathophysiologische Geschehen bislang nicht möglich ist, beschränkt sich die konservative Therapie auf eine konsequente intensivmedizinische Basistherapie.

Die Intensivtherapie der schweren, nekrotisierenden Verlaufsform beinhaltet eine Basistherapie zur Sicherung der Vitalfunktionen mit einer oralen Nahrungskarenz mit parenteraler Ernährung und einer suffizienten Analgesie. In der Frühphase des toxischen Geschehens (SIRS) richtet sich die Therapie insbesondere gegen die Hypovolämie, induziert durch die Sequestration in dritte Räume, das respiratorische Versagen, die renale Insuffizienz und metabolische Entgleisungen. In der Spätphase der schweren Pankreatitis richten sich die therapeutischen Ansätze gegen septische Komplikationen (z.B. intraabdominelle oder intrapankreatische Abszesse). Im Vordergrund stehen z.Z. konservative intensivmedizinische Therapiemaßnahmen; operative Interventionen kommen meist nur bei lokalen Komplikationen zur Anwendung (Nekrosektomie, Abszeßausräumung). Spezifische Therapieverfahren (z.B. Proteaseninhibitoren) konnten in klinischen prospektiven, randomisierten Studien nicht überzeugen. Neue Therapieansätze, insbesondere die kontinuierliche venovenöse Hämofiltration und die isovolämische Hämodilution durch Einsatz hyperonkotischer Dextranlösungen, waren in tierexperimentellen Studien erfolgversprechend. Ihre Wirksamkeit muß jedoch noch in klinischen Untersuchungen evaluiert werden.

\section{Literatur}

1. Reynaert MS, Dugernier T, Kestens PJ (1990) Current therapeutic strategies in severe acute pancreatitis. Intensive Care Med 16:352-362

2. Ranson JHC (1984) Acute pancreatitis: pathogenesis, outcome and treatment. Clin Gastroenterol 14:843-863

3. Beger HG, Bittner R, Block S, Büchler M (1988) Bacterial contamination of pancreatic necrosis. A prospektive clinical study. Gastroenterology 91:433

4. Ranson JHV (1981) Conservative surgical treatment of acute pancreatitis. World J Surg 5:351-359

5. Smadja C, Bismuth H (1986) Pancreatic debridement in acute necrotizing pancreatitis: an obsolete procedure? Br J Surg 73:408-412

6. Beger HG, Krautzberger W, Bittner R, Block S, Büchler M (1985) Results of surgical treatment of necrotizing pancreatitis. World J Surg 9:972-979

7. Rattner DW, Warshaw AL (1988) Surgical intervention in acute pancreatitis. Crit Care Med 16:89-95

8. Singer MV, Gyr K, Sarles H (1985) Revised classification of pancreatitis. Gastroenterology 89:683-685

9. Schmitz-Moormann P (1987) Akute Pankreatitis, Morphologie und Pathogenese. Verh Dtsch Ges Path 71:161-174

10. Klar E (1992) Ätiologie und Pathogenese der akuten Pankreatitis. Helv Chir Acta 59:7-16

11. Allan J, White TT (1974) An alternate mechanism for the formation of protein plugs in chronic calcifying pancreatitis. Digestion 11:428-431

12. Brugge WR, Burke CA, Brand DL, Chey WY (1985) Increased interdigestive pancreatic trypsin 
secretion in alkoholic pancreatic disease. Dig Dis Sci 30:431-439

13. Blank S, Wise L, Gersten A (1983) Risk factors in acute pancreatitis. Am J Gastroenterol 78637-642

14. Garca-Szabo RR, Malik AB (1984) Pancreatitis-induced increase in lung vascular permeability. Am Rev Respir Dis 129:580-583

15. Di Carlo V, Nespoli A, Chiesa R (1981) Hemodynamic and metabolic impairment in acute pancreatitis. World J Surg 5:329-339

16. Beger HG, Bittner R, Büchler M (1986) Hemodynamic data patterns in patients with acute pancreatitis. Gastroenterology 90:74-78

17. Schafmeyer A, Köhler H, Martell J (1989) Hämodynamische Charakteristika der nekrotisierenden Pankreatitis. Zentrbl Chir 114:107-113

18. Bittner R, Block S, Büchler M, Beger HG (1987) Pancreatic abscess and infected pancreatic necrosis - different local septic complications in acute pancreatitis. Dig Dis Sci 32:1082

19. Malfertheimer P, Büchler M, Schoetensack C (1987) Laboratory parameters for the detection of acute necrotizing pancreatitis. Digestion 38:38-42

20. Gudgeon AM, Heath DI, Hurley P, Jehanli A, Patel G (1990) Trypsinogen activation peptides assay in the early prediction of severity of acute pancreatitis. Lancet I:4-8

21. Ranson JHC, Rifkind KM, Roses DF (1974) Prognostic signs and the role of operative management in acute pancreatitis. Surg Gynecol Obstet 139:69-84

22. Silverstein W, Isikoff MB, Hill MC, Barkin J (1981) Diagnostic imaging of acute pancreatitis: prospective study using CT and sonography. AJR 137497-501

23. Clavien PA, Hauser H, Meyer P (1988) Value of contrast-enhanced computerized tomography in the early diagnosis and prognosis of acute pancreatitis. Am J Surg 155:457-466

24. Ranson JHC (1979) The timing of biliary surgery in acute pancreatitis. Ann Surg 189:654-663

25. Blamey SL, Imrie CW (1984) Prognostic factors in acute pancreatitis. Gut 25:1340-1346

26. Creutzfeld W, Lankisch PG (1981) Intensive medical treatment of severe acute pancreatitis. World $\mathbf{J}$ Surg 5:341-350

27. Staritz M, Poralla T, Manns M, Meyer zum Büschenfelde KH (1986) Effect of modern analgesic drugs (Tramadol, pentazocine, and buprenorphin) on the bile duct sphincter in man. Gut 27:265-287

28. Lankisch PG (1987) Möglichkeiten und Grenzen der konservativen Behandlung der akuten Pankreatitis. Chirurg 58:64-69

29. Niesel HC, Klimpel L, Kaiser H, Bernhardt A, AlRafai S, Lang U (1991) Epidurale Blockade zur Analgesie und Behandlung der akuten Pankreatitis. Reg Anaesth 14:97-100
30. Sitzman J, Steinborn PA, Zinner MH (1989) TPN and alternative energy substrates in the treatment of severe acute pancreatitis. Surg Gynecol Obstet 168:311-317

31. Pfisters PWT, Ranson JHC (1992) Nutritional support of acute pancreatitis. Surg Gynecol Obstet 175:275-284

32. Edelman K, Valenzuela JE (1983) Effect of intravenous lipid on human pancreatic secretion. Gastroenterology 85:1063-1066

33. Trapnell JE, Rigby CC, Talbot $\mathrm{CH}$, Duncan EHL (1974) A controlled trial of trasylol in the treatment of acute pancreatitis. Br J Surg 61:177-182

34. MRC Multicentre Trial of Glukagon and Aprotinin (1977) Death from acute pancreatitis. Lancet 632-635

35. Büchler M, Malfertheimer P, Uhl W, Stockmann F, Schölmerich J (1990) The german multicentre doubleblind randomised study of gabexate-mesilat (4 g/day IV) in acute pankreatitis. Abstract Gastroenterology 98:A214

36. Leese T, Holliday M, Watkins M, Neoptolemos JP, Thomas WM (1988) Preliminary results of of a multicentre controlled clinical trial of high-volume fresh frozen plasma therapy in prognostically severe acute pancreatitis. Digestion 4097-100

37. Lankisch PG, Otto J, Göke B, Rahlf G (1986) No effect of ranitidine on acute experimental pancreatitis. Dig Dis Sci 31:780-783

38. Cameron JL, Mehigan. D, Zuidema GD (1979) Evaluation of atropine in acute pancreatitis. Surg Gynecol Obstet 148:206-208

39. Goebell H, Ammann R, Herfarth C, Horn J, Hotz J (1979) A double-blind trial of synthetic salmon calcitonin in the treatment of acute pancreatitis. Scand J Gastroenterol 14:881-889

40. Usadel KH, Überla KK, Leuschner U (1985) Treatment of acute pancreatitis with somatostatin: results of the multicenter double-blind trial (APTS-study). Dig Dis Sci 30:992-997

41. Klar E, Herfarth C, Messmer K (1990) Therapeutic effect of isovolemic hemodilution with dextran 60 on the impairment of pancreatic microcirculation in acute biliary pancreatitis. Ann Surg 211:346-353

42. Schmidt J, del Castillo Fernandez, Rattner DW, Lewandrowsky $\mathrm{KB}$, Messmer $\mathrm{K}$, Warshaw $\mathrm{AL}$ (1993) Hyperoncotic ultrahigh molecular weight dextran solutions reduce trypsinogen activation, prevent acinar necrosis, and lower mortality in rodent pancreatitis. Am J Surg 165:40-45

43. Barzilay E, Kessler D, Berlot G, Gullo A, Geber D, Ben-Zeev I (1989) Use of extracorporal supportive techniques as additional treatment for septic-induced multiple organ failure patients. Crit Care Med 17:634-637

44. Tonnesen E, Hansen M, Höhndorf K, Bendtzen K, Diamant M (1991) Cytokines in plasma and ultra- 
filtrate in relation to hemofiltration (abstract). Anesthesiology 75:A266

45. Bellomo R, Tipping P, Boyce N (1993) Continuous veno-venous hemofiltration with dialysis removes cytokines from the circulation of septic patients. Crit Care Med 21:522-526
46. Blinzler L, Hausser J, Bödeker H, Zaune U, Martin E, Gebhardt C (1991) Conservative treatment of severe necrotizing pancreatitis using early continuous venovenous hemofiltration. Contrib Nephrol 93:234-236 